\title{
Vertex finding in ALICE by the use of silicon pixel layers in the Inner Tracking System
}

\author{
A. Badalà, R. Barbera, G. Lo Re, A. Palmeri, \\ A. Pulvirenti, G. S. Pappalardo and F. Riggi \\ for the ALICE Collaboration \\ INFN, Sezione di Catania, and Dipartimento di Fisica e Astronomia, \\ Università di Catania, Corso Italia 57, Catania (Italy)
}

\begin{abstract}
The performance of the Inner Trac king System of the ALICE detector for primary vertex estimation is discussed. A simulation study of the vertex reconstruction in ALICE through the information giv en by the tw opixel layers is reported. V ertex resolution was studied as a function of the nominal vertex location and of the particle multiplicity.
\end{abstract}

\section{Introduction}

The ALICE experiment [1] at LHC plans to build an Inner T rac kingystem (ITS) [2] made by six lay ers of silicon detectors. The aim of such detector is to improve the tracking procedure near the interaction point and to find primary and secondary vertices due to hyperon and open charm decay. The primary vertex position in ALICE is constrained to a region which can be parametrized by a Gaussian along the $\mathrm{z}$ (beam)-direction, with an RMS $\sigma_{z}$ $=5.3 \mathrm{~cm}$. The width parameter in the transverse plane $\sigma_{x, y}$ will depend on the choice of the beam parameter $\beta^{*}$, ranging from $15 \mu \mathrm{m}$ to several tens of microns. Moreover, different fillings of the LHC ring will giv eaveragebeam positions in the transverse plane which may differ by several millimeters. The information on primary vertex may be obtained by the two inner layers of pixel detectors, due to their large granularity (about $10 \mathrm{M}$ channels) and small distances $(\mathrm{r}=4$ and $7 \mathrm{~cm})$ from the interaction point. Howev er, a careful simulation study of the problem is needed in order to evaluate the performance of such a tracking device, and to correctly tune the reconstruction algorithms to improve the knowledge of the vertex location under different conditions 
Table 1

Geometrical parameters of the ITS layers.

\begin{tabular}{rrrrr}
\hline Layer & Type & $(\mathrm{cm})$ & $\pm \mathrm{z}(\mathrm{cm})$ & No. of channels \\
\hline 1 & pixel & 4.1 & 14.35 & $3279 \mathrm{~K}$ \\
2 & pixel & 7.2 & 14.35 & $6558 \mathrm{~K}$ \\
3 & drift & 14.8 & 22.2 & $43 \mathrm{~K}$ \\
4 & drift & 23.5 & 29.7 & $90 \mathrm{~K}$ \\
5 & strip & 38.5 & 45.1 & $1201 \mathrm{~K}$ \\
6 & strip & 43.6 & 50.8 & $1518 \mathrm{~K}$ \\
\hline
\end{tabular}

(high and low multiplicity events, effect of beam displacement in the trasverse plane, choice of the magnetic field setting,...).

Sect.II briefly describes the Inner Tracking System in the ALICE detector, while Sect.III is devoted to a discussion of the different aspects of the use of such system as a vertex detector.

\section{The Inner Tracking System in ALICE}

The Inner Tracking System in ALICE is made of six layers of coordinatesensitive detectors, which cover the central rapidity region $(-0.9 \leq \eta \leq+0.9)$. The geometrical parameters and the technology used in the ITS are summarized in Table 1.

The detectors and front-end electronics are held by carbon-fibre structures, which also support the cabling and cooling connections of the six ITS layers to the outside services. The granularity required for the two innermost layers is achieved with micropattern detectors based on silicon pixel sensors. These layers are essential for the vertex capabilities of ALICE. They are expected to operate with a large track density, up to $90 / \mathrm{cm}^{2}$, and in a relatively high radiation environment, of the order of $200 \mathrm{krad}$ in 10 years for the first layer.

As a result of a dedicated RD Collaboration at CERN, a final readout chip to be bump-bonded to the pixel sensor matrix, has been designed, following the development of the Omega families, still in use in the NA57 experiment at CERN SPS [3]. Each front-end chip contains the electronics for the readout of 8192 detector cells ( $32 \times 256)$; the cell size is $50 \times 425 \mu \mathrm{m}$. The front-end chips were designed with a radiation-tolerant layout technique in $0.25 \mu \mathrm{m}$ CMOS. Several irradiation tests have proven that such a design is tolerant up to a few tens of Mrad. 


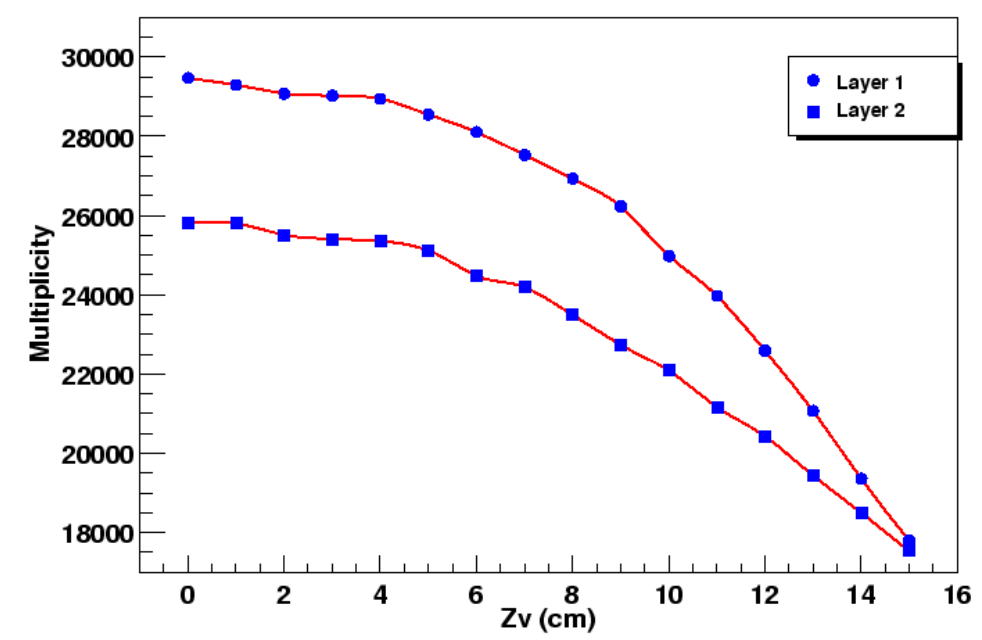

Fig. 1. Multiplicity of the reconstructed points in the two pixel layers, as a function of the primary vertex location along the beam axis. Central $\mathrm{PbPb}$ collisions were used.

\section{Reconstruction of primary vertex in ALICE}

The location of the primary vertex may be determined in ALICE by the information provided by the two inner layers of pixel detectors, looking at the correlation between the impact points of the track in the two layers. The precision to which the location of the primary vertex can be pinned down will depend on the particle multiplicity (fig.1).

A great effort has been made within the ALICE Collaboration to have a comprehensive simulation and reconstruction package, AliRoot [4], which is discussed in Sect.3.1. The basic algorithm for vertex finding is presented in Sect.3.2, together with typical results. Since the effect of the beam displacement in the transverse plane needs to be taken into account, a detailed discussion of such problem is made in Sect.3.3, with the aim of designing a full 3D vertex reconstruction procedure, and to report the obtained results. Finally, some comparison between the results expected at lower and higher magnetic field settings in ALICE is reported in Sect.3.4.

\subsection{The simulation framework}

All the simulations carried out for this study were done with the simulation and reconstruction package designed for ALICE, AliRoot Vers.3.05. Heavy-ion 
collisions were generated by the HIJING heavy ion event generator, following all the particles extracted in the full solid angle and in the full momentum range. Different vertex locations, both along the beam axis (from $-15 \mathrm{~cm}$ to $+15 \mathrm{~cm}$ ) and in the transverse plane (up to radial distances of $10 \mathrm{~mm}$ ) were used. As far as the geometry of the ALICE detector is concerned, the six layers of the ITS, the beam pipe and the magnet were included. It was verified that the inclusion of additional detectors, such as the front absorber or the Time-Projection-Chamber (TPC) does not contribute to the combinatorial background and does not alter at all the results. Particles are followed inside the experimental set-up by the standard GEANT routines, with all physical effects switched on. Full response of the pixel detectors was considered in the reconstruction process; this includes realistic values of the thresholds, charge sharing effects between neighbouring cells, and center-of-gravity evaluation of the impact point.

\subsection{Algorithm for vertex finding}

The basic method to evaluate the primary vertex location makes use of the correlation between the impact points in the two pixel layers. Different aspects of the method have been already reported within the ALICE Collaboration [58]. Referring to fig.2, a rough estimation of the vertex location, $\mathrm{z}_{v}^{0}$, is made first, using the z-distribution of the reconstructed points in the innermost pixel layer. The centroid of such distribution is well correlated with the true position of the vertex, $\mathrm{z}_{\text {true }}$, and may be used to estimate $\mathrm{z}_{v}^{0}$. The correlation of the points $\mathrm{z}_{1}, \mathrm{z}_{2}$ in the two layers is then considered, selecting only those pairs which point to a vertex position $\mathrm{z}_{v}$ within a specified confidence region around $\mathrm{z}_{v}^{0}$. An additional cut on the $\Delta \varphi$ difference between the two azimuthal angles $\varphi_{1}, \varphi_{2}$ was used to reduce the combinatorial background.

With such cuts, a distribution similar to that shown in fig.3 is obtained. A fit of such spectrum by a Gaussian peak and a constant background is able to provide an estimate of the vertex position and its uncertainty. Typical results on the performance of the method are reported in fig.4, for different vertex locations along the beam axis, as the difference between the true vertex position and the found value.

The differences are in the order of $5 \mu \mathrm{m}$, which is a value comparable with the vertex resolution, and in the same order as the statistical fluctuations from event to event. The ability to reconstruct the vertex location was also investigated for different particle multiplicities, down to a value of 17000 , which roughly corresponds to CaCa collisions. Even though the combinatorial background is reduced for low particle multiplicities, the loss in statistics results in a slightly worse vertex resolution, in the order of $10 \mu \mathrm{m}$ for medium mass 


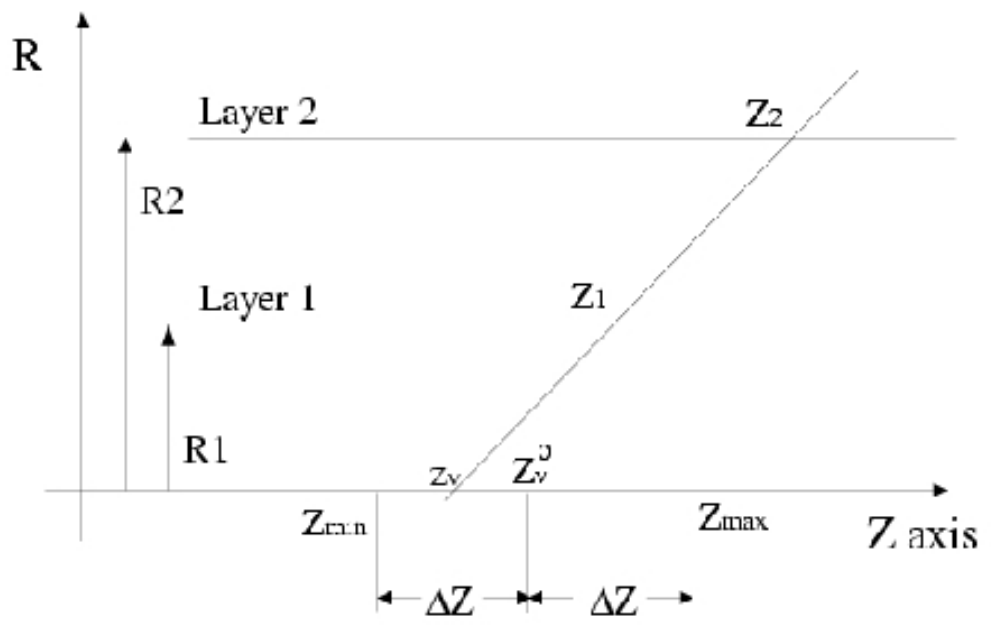

Fig. 2. Geometrical sketch of the algorithm used to correlate the points in the two pixel layers.

systems.

If the vertex resolution is plotted as a function of the particle multiplicity (fig.5), a power law

$$
\sigma_{z}=\frac{A}{\sqrt{(}\left(d N_{c h} / d \eta\right)}+B
$$

can be fitted to the data. 


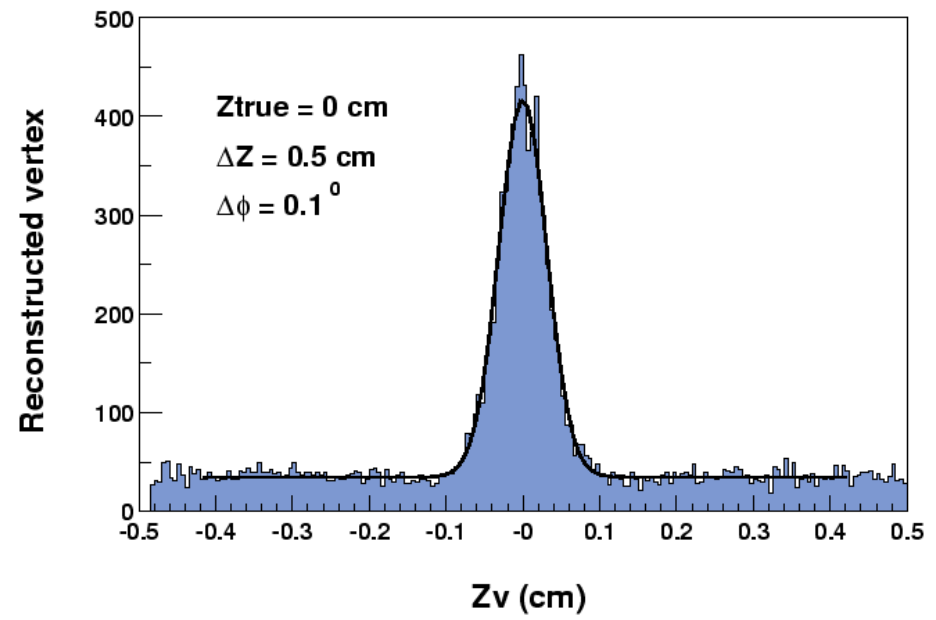

Fig. 3. Distribution of $\mathrm{z}_{v}$ for a central $\mathrm{PbPb}$ collision. The centroid of the Gaussian peak and its uncertainty may be used as an estimate of the vertex position.

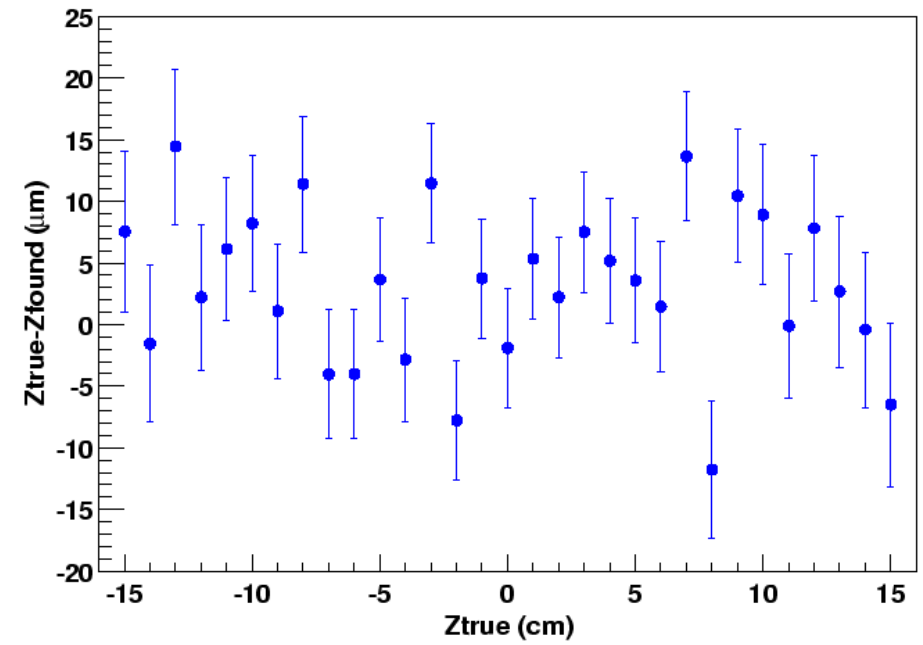

Fig. 4. Difference between the found and the true position of the vertex, as a function of the true position.

\subsection{Effect of beam displacement and 3D vertex reconstruction}

The beam displacement in the transverse plane (xy) which is expected between different fillings of the LHC ring and the intrinsic width $\sigma_{x, y}$ of the beam profile 


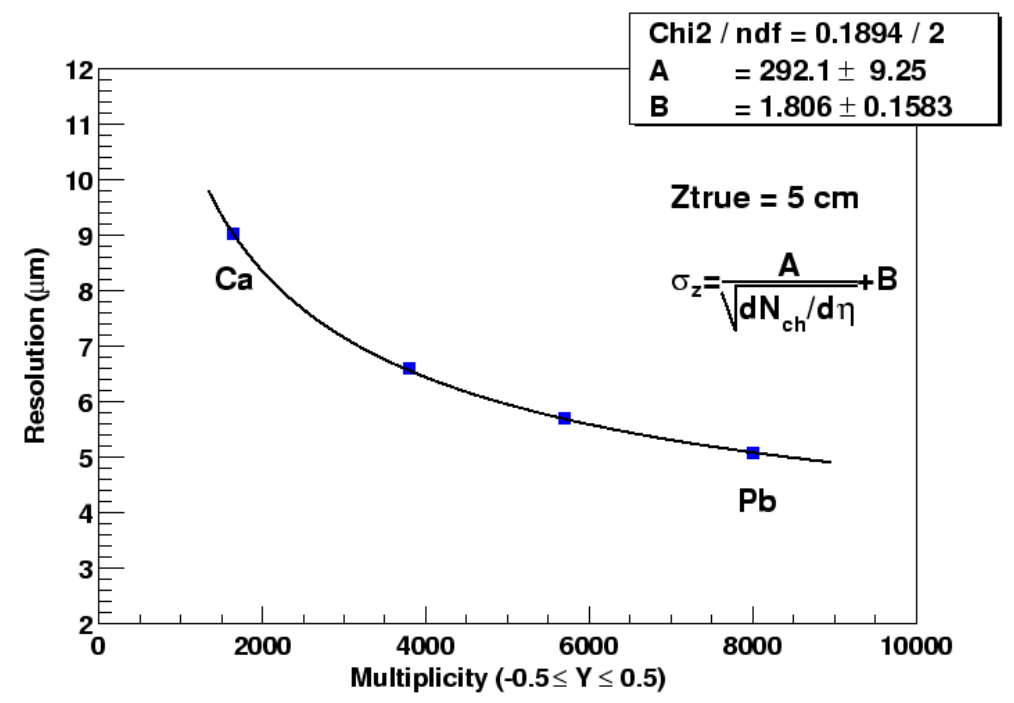

Fig. 5. Resolution in the found vertex position, as a function of the particle multiplicity. The solid line is a fit through the parametrization given in the figure.

need to be considered for a correct evaluation of the vertex location. The beam displacement off the z-axis has two mainly effects on the vertex finding: first of all, it alters the information on the $\mathrm{z}$ coordinate of the vertex, thus requiring a correction on an event-by-event basis. Secondly, especially if $\sigma_{x, y}$ is not negligible, it introduces the requirement of a full 3D vertex reconstruction. The $(\mathrm{x}, \mathrm{y})$ vertex coordinates could actually be found by summing several events altogether, since in each filling such position will only change within a range governed by $\sigma_{x, y}$ from event to event. However, for large values of $\sigma_{x, y}$ it will be preferable to evaluate the $3 \mathrm{D}$ position of the vertex in each individual event. This has been done using a first estimate of the vertex location in the transverse plane, from the upper, lower, left and right (looking at the beam) multiplicities, and introducing such estimate in the evaluation of the $\mathrm{z}$ coordinate. With such a correction, an optimal vertex resolution $(5 \mu \mathrm{m})$ is preserved even for beam displacement up to $10 \mathrm{~mm}$, whereas neglecting such a correction, the resolution increases dramatically to about $200 \mu \mathrm{m}$ as soon as the beam is shifted by only $2 \mathrm{~mm}$.

In the transverse plane the correlation of the points generated by a track in the two pixel layers cannot be strictly done through a straight line, as for the z-axis, due to the magnetic field. However, the deviation from a straight line is small and a reasonable result may be expected, especially for particles with high momentum. In order to improve the performance of the method, an iterative procedure has been designed [8]. With such a procedure it was possible to get the results shown in fig.6. 

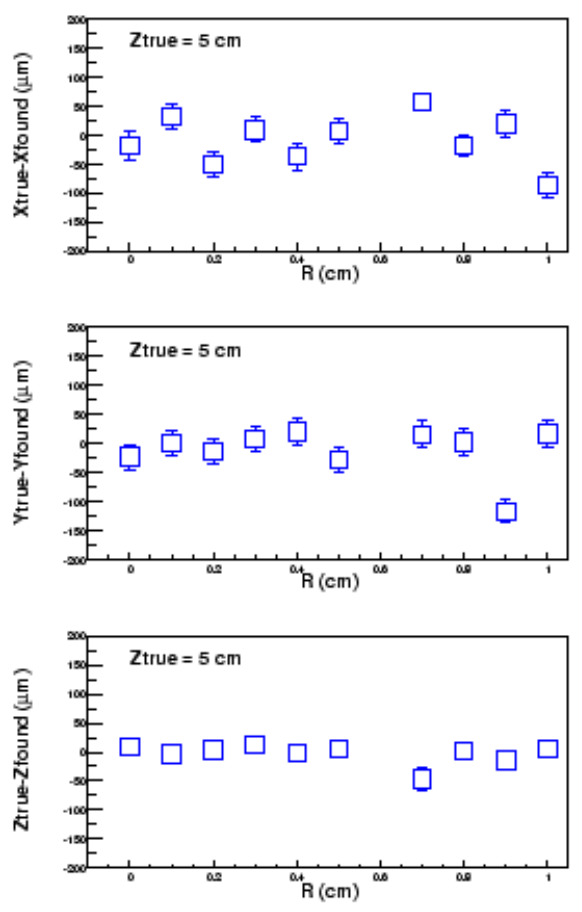

Fig. 6. Differences between the true and found values of the $x, y, z$ coordinate of the vertex position, as a function of the radial distance from the beam axis.

As it is seen, the resolution is still in the order of a few $\mu \mathrm{m}$ along the z-axis, while it is a little bit poorer (around $25 \mu \mathrm{m}$ ) - as expected - along $\mathrm{x}$ and y. Such values are still very good for most of the physics to be done with the ITS in ALICE. As an example, this precision makes it possible the study of strangeness production $\left(\mathrm{K}_{s}^{0}, \Lambda, \Xi, \Omega\right)$ which needs to identify secondary vertices and their impact parameter with respect to the primary vertex.

\subsection{Choice of magnetic field}

All the simulations performed so far were done by the commonly assumed value of the magnetic field in ALICE, i.e. $B=0.2 \mathrm{~T}$. On-going discussions within the ALICE Collaboration have shown that larger values of the field (up to $B=0.5$ $\mathrm{T}$ ) could be used for some physics topics of interest. In view of such a choice, the performance of the overall method was checked as a function of the field 
intensity. For higher magnetic field settings, two main effects are expected: the first is a possible increase of the occupancy on the first two layers, due to low momenta particles, which could increase the combinatorial background and hence worsen the resolution. It was verified however that the relative increase in the occupancy is only a few percent. Another effect comes from the straight line approximation in the transverse plane, which is less and less good as B increases, thus requiring to choose a larger $\Delta \varphi$ cut to account for a larger uncertainty in the individual azimuthal angles.

The overall result of such effects is to increase the vertex resolution from 5 $\mu \mathrm{m}$ to $7-8 \mu \mathrm{m}$ along $\mathrm{z}$, and from $25 \mu \mathrm{m}$ to $30-35 \mu \mathrm{m}$ along $\mathrm{x}, \mathrm{y}$, when the field goes from $0.2 \mathrm{~T}$ to $0.5 \mathrm{~T}$.

\section{Conclusions}

We have carried out a complete study of the vertex finding problem in the general case of a primary vertex located outside the z-axis. A full 3D algorithm has been designed and tested as a part of the reconstruction procedure, using the information from the two innermost (pixel) layers. The full response of the pixel detectors was included in the simulations. The $\mathrm{x}, \mathrm{y}$ and $\mathrm{z}$ coordinates of the vertex were found by the correlation of the reconstructed points in the two pixel layers, with proper cuts on the $\Delta \phi$ difference and on the fiducial region around the first estimate of the vertex position. The results show that it is possible to achieve a very good resolution, in the order of $5 \mu \mathrm{m}$ along the z-axis, even in case of beam displacement off the z-axis, up to radial distances of $10 \mathrm{~mm}$. The $(\mathrm{x}, \mathrm{y})$ coordinates of the vertex are found within $25 \mu \mathrm{m}$, due to the intrinsic limitation of the straight line approximation in the transverse plane. The choice of a high magnetic field setting, such as $B=0.5 \mathrm{~T}$, was seen not to dramatically alter the performance of the method, only increasing the resolution from 25 to $30-35 \mu \mathrm{m}$ in the transverse plane. All these results may be obtained on an event-by-event basis, which could be important especially in case a large value of the $\beta^{*}$ parameter is chosen, which in turn gives a large width of the Gaussian profile along $\mathrm{x}, \mathrm{y}$.

A good knowledge of the primary vertex will be essential for the recognition of secondary vertices due to the decay of particles with a low value of $\mathrm{c} \tau$, such as the D mesons. It will be also of great importance for a correct estimation of the impact parameter of the tracks originating from the decay of (multi)strange baryons. 


\section{References}

[1] ALICE Technical Proposal, CERN/LHCC 95-71 (1995).

[2] ITS Technical Design Report, CERN/LHCC 99-12(1999).

[3] The NA57 Collaboration, Nucl. Physics A681(2001)165.

[4] For the AliRoot Package see the following address: http://alisoft.cern.ch/offline/aliroot-pro/manual.html

[5] J.Hubbel, B.Nilsen and L.Vannucci, Internal Note ALICE-2000-10.

[6] N.Bustreo et al., Internal Note ALICE-INT-2001-13.

[7] A.Badalà et al., Internal Note ALICE-INT-2001-11.

[8] A.Badalà et al., Internal Note ALICE-INT-2001, submitted. 